\title{
CONVEXITÉ HOLOMORPHE INTERMÉDIAIRE DES REVETEMENTS D'UN DOMAINE PSEUDOCONVEXE
}

\author{
S. Asserda
}

Let $M$ be a complex manifold and $L \mapsto M$ be a positive holomorphic line bundle over $M$ equipped with a Hermitian metric $h$ of class $C^{2}$. If $D \subset \subset M$ is a pseudoconvex domain which is relatively compact in $M$ then there exists an integer $r_{0}$ such that for every $r \geqslant r_{0}$ and for every connected holomorphic covering $\pi$ : $\widetilde{D} \rightarrow D$, the covering $\widetilde{D}$ is holomorphically convex with respect to holomorphic sections of $\left(\pi^{*}\left(L_{D}^{r}\right), \pi^{*} h^{r}\right)$.

\section{INTRODUCTION ET ÉNONCÉ DU RÉSULTAT PRINCIPAL}

Un domaine $D \subset \subset \mathbf{C}^{n}$ est dit localement pseudoconvexe si tout point $x_{0} \in \partial D$ admet un voisinage $U_{x_{0}} \subset \mathbf{C}^{n}$ tels que $U_{x_{0}} \cap D$ soit pseudoconvexe. On adopte une definition analogue pour les domaines dans les variétés analytiques complexes.

DÉFINITION 1.1: Un domaine $D$ relativement compacte dans une variété analytique complexe $M\left(\operatorname{dim}_{c} M=n\right)$ est localement pseudoconvexe si pour tout point $x_{0} \in \partial D$ il existe une carte locale $\left(U_{x_{0}}, \Psi_{x_{0}}\right)$ passant par $x_{0}$ tels que $\Psi_{x_{0}}\left(U_{x_{0}} \cap D\right)$ soit pseudoconvexe dans $\mathbf{C}^{n}$.

Dans [4] Grauert introduit la notion de convexité holomorphe par rapport aux sections holomorphes d'un fibré vectoriel, qui généralise la convexité holomorphe ordinaire.

Définition 1.2: Soient $N$ une variété analytique complexe, $L$ un fibré vectoriel holomorphe au dessus de $N$ et $h$ une metrique Hermitienne de classe $C^{2}$ sur $L$. La variété $N$ est dite $L$-convexe si pour toute partie infinie $S \subset N$ sans point d'accumulation dans $N$, il existe une section holomorphe $\sigma$ de $L$ sur $N$ telle que la fonction $\|\sigma(?)\|_{h}$ soit non bornée sur $S$.

REMARQUES.

(i) Si $L$ est de rang un et $D \subset \subset N$ est $L$-convexe alors $D$ est localement pseudoconvexe.

(ii) Si $D \subset \subset N$ alors la notion de $L$-convexité est indépendante du choix de la métrique $h$ sur $L$.

Received 18th November, 1996.

Research supported by a grant of MES.FC.RS : SC-2.17033 at FSK 1.11.95.

Copyright Clearance Centre, Inc. Serial-fee code: 0004-9729/97 \$A2.00+0.00. 
La restriction de $L$ sur $D$ est notée par $L_{D}$ et $\otimes^{r} L$ désigne le produit tensoriel de $L$ ( $r$ fois).

Dans [1] le résultat suivant est démontré :

THÉorème 1.1. Soient $M$ une variété analytique complexe et $(L, h)$ un fibré en droites holomorphe positif sur $M$. Si $D \subset \subset M$ est un domaine localement pseudoconvexe et relativement compact dans $M$, alors il existe un entier $r_{0}$ tel que $D$ soit $\otimes r L$-convexe poir tout $r \geqslant r_{0}$.

Soient $M, D,(L, h)$ comme dans le théorème précédent et $\widetilde{D}$ un revetement holomorphe connexe de $D$. Il est naturel de se demander si $\widetilde{D}$ est $\left(\pi^{*}\left(L_{D}^{r}\right), \pi^{*} h^{r}\right)$ convexe pour $r$ assez grand. Dans cette note on démontre le résultat suivant:

THÉoRème 1.2. Supposons que $(L, h)$ soit positif sur $M$ et que $D \subset \subset M$ est localement pseudoconvex et relativement compact dans $M$. Alors il existe un entier $r_{0}$ tel que pour tout $r \geqslant r_{0}$ et tout revetement holomorphe connexe $\pi: \widetilde{D} \rightarrow D$, le revêtement $\widetilde{D}$ est $\left(\pi^{*}\left(L_{D}^{r}\right), \pi^{*}\left(h^{r}\right)\right)$-convexe.

Rappelons qu'un fibré holomorphe en droites $L$ est dit positif sur $M$ si la $(1,1)$ forme de courbure $c(L)_{h}$ est définie positive sur le fibré tangent holomorphe $T M$ de $M$. Si $(U, \Psi)$ est une trivialisation de $L$ sur $U, L_{U}=\pi^{-1}(U) \longmapsto U \times \mathbf{C}$, alors la métrique $h$ de $L$ est donnée par

$$
\|\xi\|_{h}=|\xi| e^{-\psi(x)} \quad \forall x \in U, \quad \forall \xi \in L_{x} \simeq \mathbf{C}
$$

et $c(L)_{h}$ s'écrit

$$
c(L)_{h}=i \partial \bar{\partial} \psi \quad \text { sur } \quad U
$$

où $\psi$ est une fonction de classe $C^{2} \operatorname{sur} U$. Si $(V, \Phi)$ est une autre trivialisation alors $\psi=\phi+\log \left|\Psi \circ \Phi^{-1}\right| \quad$ sur $\quad U \cap V$ and $i \partial \bar{\partial} \log \left|\Psi \circ \Phi^{-1}\right|=0$. Donc $c(L)_{h}$ est définie positif sur $U$ si et seulement si $\psi$ est une fonction strictement plurisousharmonique.

Si $u$ et $v$ sont des $(p ; q)$-formes sur $M$ à valeurs dans $L$, la quantité $\langle u, v\rangle_{h}$ désigne leur produit par rapport à $h$ et une metrique donnée $g$ sur $M$. L' adjoint formel de l'operateur $\bar{\partial}$ sur $L$ est noté $\bar{\partial}^{*}$ et Ricci $(g)$ désigne la courbure de Ricci de $g$. Pour de plus amples détails voir $[\mathbf{3}, \mathbf{5}]$

\section{DÉMONSTRATION DU THÉORÈme 1.2.}

Soit $S$ une partie infinie de $\widetilde{D}$ sans point d'accumulation. On construit une section holomorphe $\sigma$ de $\pi^{*}\left(L_{D}^{r}\right)$ au dessus de $\widetilde{D}$ telle que $\|\sigma(?)\|_{\pi^{*} h^{r}}$ soit non bornée sur $S$. Puisqu'il suffit de considérer chaque partie infinie de $S$, on peut supposer que $S$ est égale à une suite de points $\left\{z_{\nu}\right\}$. On pose $x_{\nu}:=\pi\left(z_{\nu}\right)$.

Si la suite $\left\{x_{\nu}\right\}$ n'admet pas de point d'accumulation dans $D$, d'après le Théorème 1.1 , pour $r \geqslant r_{0}$ assez grand $\left(r_{0}\right.$ ne depend que de $\left.D\right)$, il existe une section holomorphe 
$s$ de $L^{r}$ sur $D$ telle que $\|s\|_{h^{r}}$ soit non bornée sur $\left\{x_{\nu}\right\}$. La section holomorphe $\sigma:=\pi^{*} s$ de $\left(\pi^{*}\left(L_{D}^{r}\right), \pi^{*}\left(h^{r}\right)\right)$ sur $\widetilde{D}$ est donc non bornée sur $\left\{z_{\nu}\right\}$.

Si $\left\{x_{\nu}\right\}$ admet un point d'accumulation $w$ dans $D$, alors on peut supposer que $x_{\nu} \rightarrow w \in D$. Soit $g$ une métrique kählérienne complète sur $D$ [1, Proposition 1.2] et $\tilde{g}=\pi^{*} g$ l'image réciproque de $g$. Puisque $\pi$ est un revetement holomorphe, $\tilde{g}$ est une metrique kählérienne complète sur $\widetilde{D}$. Soit $(V, \Psi) \simeq B(0,2 R)$ une carte locale centrée en $w$ et de rayon $2 R$ telle que $L_{V}$ soit trivial. Soit $t$ une section holomorphe de $L^{r}$ sur $M$ telle que $t(w) \neq 0$. Quitte à prendre $R$ assez petit, il existe un voisinage $\mathcal{O}$ de $Y=\{x \in D ; t(x)=0\}$ dans $(D, g)$ telle que $\bar{V} \cap \overline{\mathcal{O}}=\emptyset$.

On pose $U:=\Psi^{-1}(\mathcal{B}(0, R))$. Puisque $\pi$ est un revetement, quitte à prendre $R$ assez petit, on peut écrire $\pi^{-1}(U)=\bigcup_{\nu} U_{\nu}$ avec $x_{\nu} \in U_{\nu}$ pour $\nu$ assez grand et les voisinages $U_{\nu}$ sont disjoints deux à deux et $\pi: U_{\nu} \rightarrow U$ est biholomorphe. Soit $\Psi_{\nu}: U_{\nu} \rightarrow B(0,2 R) \subset \mathbf{C}^{n}$ définie par

$$
\tilde{\Psi}_{\nu}(z):=\Psi(\pi(z))-\Psi\left(\pi\left(z_{\nu}\right)\right) \quad z \in U_{\nu}
$$

L'application $\widetilde{\Psi}_{\nu}$ vérifie les propriétés suivantes:

(1) $\widetilde{\Psi}_{\nu}\left(z_{\nu}\right)=0$ pour chaque $\nu$

(2) Il existe des constantes $a$ et $b$ indépendantes de $\nu$ telles que: $a \widetilde{\Psi}_{\nu}^{*} g_{e} \leqslant$ $\widetilde{\Psi}_{\nu}^{*} g \leqslant b \widetilde{\Psi}_{\nu}^{*} g_{e}$ sur $U_{\nu}$, où $g_{e}$ est la métrique euclidienne.

Soit $\lambda$ une fonction test à support dans $B(0, R)$ telle que $\lambda=1$ dans un voisinage de 0 et $0 \leqslant \lambda \leqslant 1$. La fonction $\Phi: \widetilde{D} \rightarrow[-\infty,+\infty[$ définie par:

$$
\Phi(z)= \begin{cases}2 n \lambda(z) \log \left|\widetilde{\Psi}_{\nu}(z)\right| & \text { si } z \in \bigcup_{\nu} U_{\nu} \\ 0 & \text { ailleurs }\end{cases}
$$

est $C^{\infty} \operatorname{sur} \widetilde{D} \backslash\left\{z_{\nu}\right\}_{\nu=1}^{\infty}$ et d'après (2)

$$
i \partial \bar{\partial} \Phi \geqslant-K g \quad \text { au sens des courants }
$$

où $K$ est une constante positive. De plus $e^{-\Phi}$ n'est pas sommable au voisinage de $z_{\boldsymbol{\nu}}$.

Soit $\xi$ une section holomorphe de $L \operatorname{sur} V$ telle que $t(w) \neq 0$. On considère la section $s$ de $\pi^{*}\left(L_{D}\right)$ sur $\widetilde{D}$ définie par

$$
s(z)= \begin{cases}\chi\left(\widetilde{\Psi}_{\nu}(z)\right) e^{r\left(z_{\nu}\right)} \xi(\pi(z)) & \text { si } z \in \bigcup_{\nu} U_{\nu} \\ 0 & \text { ailleurs. }\end{cases}
$$

où $r(z):=d_{\tilde{g}}\left(z_{0}, z\right)$ est la distance par rapport à la métrique $\tilde{g}$ entre $z$ et un point fixé $z_{0}$ de $\widetilde{D}$. On peut choisir la fonction test $\chi$ de sorte que la $(0,1)$-forme lisse 
$\alpha:=\bar{\partial} s$ à valeurs dans $\pi^{*}\left(L_{D}\right)$ dont le support est contenu dans $\bigcup_{\nu} U_{\nu}$, s'annule dans un voisinage $\left\{\left(z_{\nu}\right)\right\} \cup \mathcal{O}$. D'après $(2)$ on a $\left|\bar{\partial}\left(\chi o \Psi_{\nu}\right)\right|_{\tilde{g}} \leqslant C$ sur $U_{\nu}$, où la constante $C$ est indépendante de $\nu$. D'où

$$
\|\alpha\|_{h^{r}}^{2} e^{-\Phi} \leqslant C^{\prime} e^{2 r\left(z_{\nu}\right)}
$$

Lemma 2.2. [7, Lemma 1.1]. Il existe une fonction $\rho: \widetilde{D} \rightarrow \mathbf{R}$ de classe $C^{\infty}$ et exhaustive vérifiant

(a) $C_{1} r \leqslant \rho \leqslant C_{2} r$

(b) $|i \partial \bar{\partial} \rho|_{\tilde{g}} \leqslant C_{3}$

dans $\pi^{-1}(U)$, où les constantes dépendent de $U$.

Soit $\left(\pi^{*} L_{D}, \pi^{*} h^{\prime}\right)$ le fibré en droites $\pi^{*}\left(L_{D}\right)$ muni de la métrique singulière $h^{\prime}:=$ $e^{-\kappa} \pi^{*} h$ où $\kappa=3 C_{1}^{-1} \rho+\Phi+\tau-\log \left\|\pi^{*} t\right\|_{\pi^{*} h^{r}}$ et $\tau:=\pi^{*} \log V_{g} / V_{M}$ ( $V_{M}$ est la forme volume associée à la métrique kählérienne $c(L)$ sur $M)$. Puisque $\tau \geqslant C_{5} \operatorname{sur} \pi^{-1}(U)$ et $\|\xi\|_{h}$ (resp $\|t\|_{h^{r}}$ ) est bornée sur $U$, on a d'après $(* *)$

$$
\int_{\widetilde{D}}\|\alpha\|_{h^{\prime}}^{2} d V_{\tilde{g}} \leqslant C^{\prime} \sum_{\nu} \int_{U_{\nu}} e^{2 r\left(z_{\nu}\right)-3 C_{1}^{-1} \rho} d V_{\tilde{g}}
$$

On peut choisir $R$ suffisamment petit de sorte que $U_{\nu} \subset B_{\tilde{g}}\left(z_{\nu}, R\right)$ pour tout $\nu$ et que les volumes des boules $B_{\tilde{g}}\left(z_{\nu}, R\right)$ soient uniformément bornés en $\nu$. Pour $z \in U_{\nu}$ on a $r\left(z_{\nu}\right)-R \leqslant r(z) \leqslant C_{1}^{-1} \rho(z)$. D'où

$$
\int_{\widetilde{D}}\|\alpha\|_{h^{\prime}}^{2} d V_{\tilde{g}} \leqslant C^{\prime \prime} \sum_{\nu} e^{-r\left(z_{\nu}\right)}<\infty
$$

car on peut supposer que $r\left(z_{\nu}\right) \geqslant \nu$ pour $\nu$ assez grand.

Soit $f$ une $(0,1)$-forme lisse sur $\widetilde{D}$ à valeurs dans $\pi^{*}\left(L_{D}\right)$. D'après l'inégalité de Cauchy-Schwarz, on a

$$
\left(\int_{\widetilde{D}}\left|\langle f, \alpha\rangle_{h^{\prime}}\right| d V_{\tilde{g}}\right)^{2} \leqslant \int_{\widetilde{D}}\|\alpha\|_{h^{\prime}}^{2} d V_{\tilde{g}} \int_{\pi^{-1}(U)}\|f\|_{h^{\prime}}^{2} d V_{\tilde{g}}
$$

Il existe des constantes $c>0$ and $d$ telles que $c(L) \geqslant c . g$ et $\operatorname{Ricci}(c(L)) \geqslant d . g$ sur $U$. Puisque $-i \partial \bar{\partial} \log \|t\|=r c(L)$ sur $U$, pour $r$ assez grand on a

$$
\operatorname{Ricci}(\tilde{g})+i \partial \bar{\partial} \Phi+i 3 C_{1}^{-1} \partial \bar{\partial} \rho+r \pi^{*} c\left(L_{D}\right)+\pi^{*} c\left(L_{D}\right)+i \partial \bar{\partial} \tau \geqslant c \tilde{g} \quad \text { sur } \quad \pi^{-1}(U) .
$$

En utilisant l'identité de Bochner-Kodaira-Nakano en géomètrie Kählerienne [2] et $(* * *)$, on déduit

$$
\left(\int_{\widetilde{D}}\left|\langle f, \alpha\rangle_{h^{\prime}}\right|^{2} d V_{\tilde{g}}\right)^{2} \leqslant \frac{1}{c} \int_{\widetilde{D}}\|\alpha\|_{h^{\prime}}^{2} d V_{\tilde{g}}\left(\int_{\widetilde{D}}\left\|\bar{\partial}^{*} f\right\|_{h^{\prime}}^{2} d V_{\tilde{g}}+\int_{\widetilde{D}}\|\bar{\partial} f\|_{h^{\prime}}^{2} d V_{\tilde{g}}\right) .
$$


Puisque $\bar{\partial} \alpha=0$, il suffit de considérer les formes $f \bar{\partial}$-fermées. Donc pour tout $f \in$ $\operatorname{Dom}\left(\bar{\partial}^{*}\right)$, on a

$$
\int_{\widetilde{D}}\left|\langle f, \alpha\rangle_{h^{\prime}}\right|^{2} d V_{\tilde{g}} \leqslant \frac{1}{c}\left(\int_{\widetilde{D}}\|\alpha\|_{h^{\prime}}^{2} d V_{\tilde{g}}\right)^{1 / 2}\left(\int_{\widetilde{D}}\left\|\bar{\partial}^{*} f\right\|_{h^{\prime}}^{2} d V_{\widetilde{D}}\right)^{1 / 2}
$$

La métrique $\widetilde{g}$ est kählérienne complète sur $\widetilde{D}$, d'après la théorie de Hörmander [5] (version singulière [2]). Il existe une section localement intégrable $\beta$ de $\pi^{*}\left(L_{D}\right)$ sur $\widetilde{D}$ telle que

$$
\bar{\partial} \beta=\bar{\partial} s \quad \text { et } \quad \int_{\widetilde{D}}\|\beta\|_{h^{\prime}}^{2} d V_{\tilde{g}} \leqslant \int_{\widetilde{D}}\|\alpha\|_{h^{\prime}}^{2} d V_{\tilde{g}}
$$

Puisque $e^{-\Phi}$ n'est pas sommable au voisinage de $z_{\nu}$ on a $\beta\left(z_{\nu}\right)=0$. La section holomorphe $\sigma:=s-\beta$ de $\pi^{*}\left(L_{D}\right)$ sur $\tilde{D}$ vérifie $\left\|\sigma\left(z_{\nu}\right)\right\|=e^{r\left(z_{\nu}\right)} \longrightarrow \infty$. Ce qui achève la démonstration du théorème.

En utilisant les techniques précedentes et l'identité de Bochner-Kodaira-Nakano en géométrie Hermitienne [3], on peut démontrer le théorème suivant qui généralise celui de Stein $[8]$ sur les revetement des variétés de Stein.

THÉORÈME 2.3. Soient $(X, g)$ une variété Hermitienne complète et $(L, h)$ un fibré holomorphe en droites positif au dessus de $X$. Si $X$ est $L$-convexe alors il existe un entier $r_{0}$ tel que pour tout $r \geqslant r_{0}$ et tout revetement holomorphe connexe $\pi: \tilde{X} \rightarrow X$, le revetement $\tilde{X}$ est $\left(\pi^{*} L^{r}, \pi^{*} h^{r}\right)$-convexe.

Remarque. Le Théorème 1.2 a été démontré par Napier [6] en supposant que la frontière de $D$ est de classe $C^{4}$.

\section{BIBLIOGRAPHIE}

[1] S. Asserda, 'The Levi problem on projective manifolds', Math $Z$. 219 (1995), 631-636.

[2] J.P. Demailly, 'Estimations $L^{2}$ pour l'opérateur $\bar{\partial}$ d'un fibré holomorphe semi-positif au dessus d'une variété kählérienne complète', Ann. Sci. Écol. Norm. Sup. 15 (1982), $457-511$.

[3] J.P. Demailly, Analytic geomety, (to appear).

[4] H. Grauert, 'Bemerkenswerte pseudokonvexe mannifaltigkeiten', Math. Z. 81 (1963), $377-392$.

[5] L. Hörmander, An introduction to complex analysis in several variables, North-Holland Mathematical Library, (Third Edition - revised), 1990.

[6] T. Napier, 'Convexity properties of coverings of smooth projective varieties', Math. Ann. 286 (1990), 433-479.

[7] T. Napier, 'Covering spaces of families of compact Riemann surfaces', Math. Ann. 294 (1992), 523-549. 
[8] K.Stein, 'Uberlayerungen holomorph-vollstandiger komplexer Râume', Arch. Math. 7 (1956), 354-361.

Université Ibn Tofail

Departement de Mathématiques

BP 133 Kénitra

Maroc 